\title{
Biomarker Discovery and Validation in Kidney Transplantation
}

\author{
Valeria R Mas* and Daniel G Maluf \\ Translational Genomics Transplant Laboratory, Transplant Division, Department of Surgery, University of Virginia, Charlottesville, VA, USA
}

Kidney transplantation (KT) is the treatment of choice for endstage kidney disease. However, the progress in short term outcomes did not translate into better long-term graft survival. Late failure of the graft continues to be a major problem after KT, in spite of more potent immunosuppressive strategies and the focus of clinical management shifting toward prolonging long-term graft survival [1]. A major challenge in clinical transplantation is to improve long-term graft survival. The biological mechanisms underlying the lack of correlation between reduced rates of acute rejection and graft survival are not currently understood. This statement emphasizes the complexity of the interactions (immunological and non-immunological) that occurs since the transplant procedure that might lead to chronic graft injury and consequently graft function loss.

A major obstacle in the management of transplant recipients is a lack of accurate and specific tests for continuous graft function monitoring as well as predicting long-term graft function [2]. Currently available and accessible methods for evaluating kidney function are either inaccurate or invasive [2,3]. The identification of the causal factors conducting to decrease in graft function might represent the best strategy to interfere in the course of progression to chronic allograft dysfunction in kidney transplant recipients.

Moreover, as consequence of the multifactorial nature of graft injury, it is highly likely that multiple parameters and markers will need to be evaluated together when assessing progression to chronic graft loss $[3,4]$. As the injury starts early post-transplantation, at the time of organ procurement and followed by ischemia-reperfusion injury, biomarkers to identify those kidney graft with poor function need to be tested early in the process when injury can be stopped or even reverse.

As a consequence of the availability of human genome data and the new technology supporting advances in genomics, proteomics and metabolomics techniques, translational research investigators have great prospects to ascertain biomarkers for various health conditions, including organ transplantation [5]. The need for better diagnostic and prognostic tools to monitor kidney graft status, as well as treatment progression and effectiveness, remains one of the most critical requirements in the field.
An ideal biomarker would be able to predict outcome onset and the harshness of specific events, such as progression to chronic allograft dysfunction even before the injury is evident at the histological level, as well as predict the specific allograft damage related to the toxicity associated with the use of immunosuppressant treatment. Regardless of the important advances attained so far in the identification of several potentially useful biomarkers of tolerance [6], organ quality [7], rejection and progression to graft dysfunction [3,4], validation and demonstration of their clinical utility still needs to be performed.

Principal limitations associated to the actual gap between biomarker discovery and clinical application in the kidney transplant setting relate to the variation among technologies (i.e., different microarray platforms), sample types (i.e., allograft tissues, peripheral blood), phenotypic variation of study groups, limited sample sizes and independent validation studies. Ultimately, in order to expedite the achievement of such important goals, all efforts should probably be performed in the context of multidisciplinary and multi-institutional supportive networks.

\section{References}

1. Meier-Kriesche HU, Schold JD, Srinivas TR, Kaplan B (2004) Lack of improvement in renal allograft survival despite a marked decrease in acute rejection rates over the most recent era. Am J Transplant 4: 378-383.

2. Bestard O, Cruzado JM, la Franquesa M, Grinyó JM (2010) Biomarkers in renal transplantation. Curr Opin Organ Transplant 15: 467-473.

3. Mas VR, Archer KJ, Scian M, Maluf DG (2010) Molecular pathways involved in loss of graft function in kidney transplant recipients. Expert Rev Mol Diagn 10: $269-284$.

4. Mannon RB (2010) Immune monitoring and biomarkers to predict chronic allograft dysfunction. Kidney Int Suppl: S59-S65

5. Perkins D, Verma M, Park KJ (2011) Advances of genomic science and systems biology in renal transplantation: a review. Semin Immunopathol 33: 211-218.

6. Roedder S, Gao X, Sarwal MM (2012) The pits and pearls in translating operational tolerance biomarkers into clinical practice. Curr Opin Organ Transplant 17: 655-662.

7. Mueller TF, Solez K, Mas V (2011) Assessment of kidney organ quality and prediction of outcome at time of transplantation. Semin Immunopathol 33: 185199

*Corresponding author: Valeria R Mas, Director and Associate Professor, Translational Genomics Transplant Laboratory, Transplant Division, Department of Surgery, University of Virginia, Charlottesville, VA 23220, USA, E-mail: VRM3N@hscmail.mcc.virginia.edu

Received November 21, 2012; Accepted November 21, 2012; Published November 23, 2012

Citation: Mas VR, Maluf DG (2013) Biomarker Discovery and Validation in Kidney Transplantation. J Mol Biomark Diagn 4:e115. doi:10.4172/2155-9929.1000e115

Copyright: @ 2013 Mas VR, et al. This is an open-access article distributed under the terms of the Creative Commons Attribution License, which permits unrestricted use, distribution, and reproduction in any medium, provided the original author and source are credited 